\title{
RAPID RECOGNITION AND ISOLATION OF LIVE COLON CANCER STEM CELLS BY USING METABOLIC LABELING OF AZIDO SUGAR AND MAGNETIC BEADS
}

\author{
Lingbo Sun, Hongxia Fu, Yanru Li, Xinrui Duan*, Zhengping Li*
}

Key Laboratory of Applied Surface and Colloid Chemistry

Ministry of Education, Key laboratory of analytical chemistry for life science of Shaanxi

Province, School of Chemistry and Chemical Engineering, Shaanxi Normal University, Xi'an, Shaanxi, 710119, P. R. China

E-Mail: duanxr@snnu.edu.cn; lzpbd@snnu.edu.cn

Table of content:

Figure $\mathrm{S}_{1}-\mathrm{S}_{4}$

S2

Figure $\mathrm{S}_{5}$ 

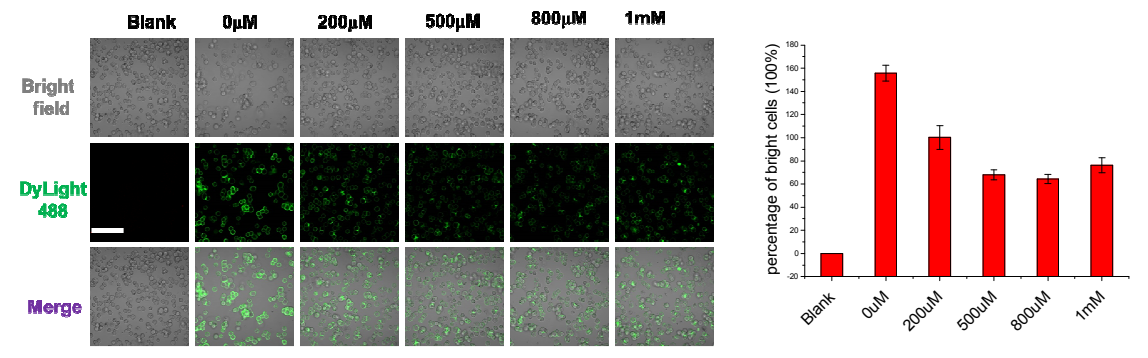

Figure S1. Optimization of the Ac4ManNAc concentration for $24 \mathrm{~h}$ incubation time. a) Fluorescence microscopy images of HCT-116 cells; b) Percentage of bright cells from five fluorescence images and total more than 900 cells were counted. Scale bar is 120 microns.
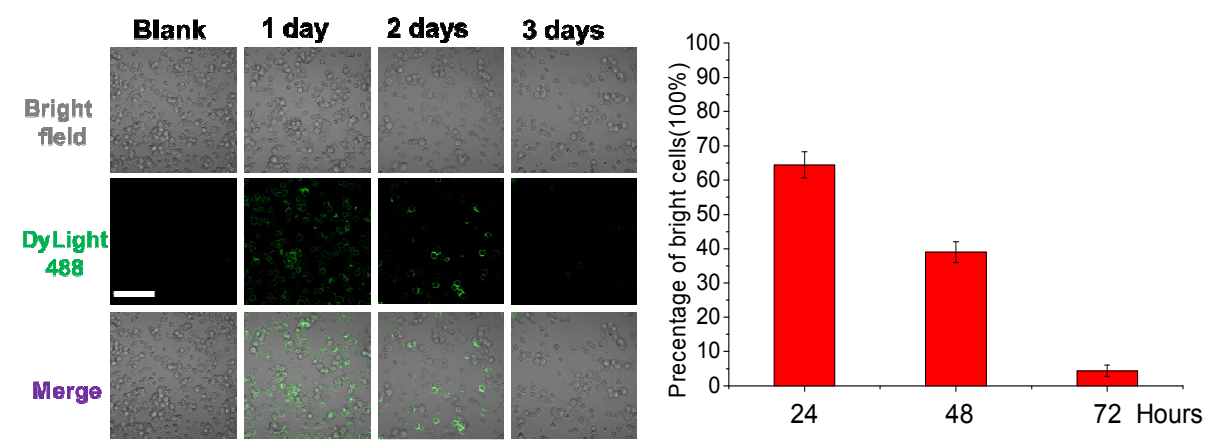

Figure S2. Optimization of the Ac4ManNAc treating time $(800 \mu \mathrm{M})$. a). Fluorescence microscopy images of HCT-116 cells; b) Percentage of bright cells from five fluorescence images and total more than 900 cells were counted. Scale bar is 120 microns.
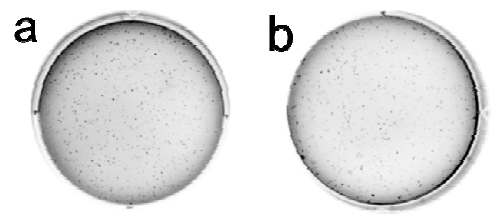

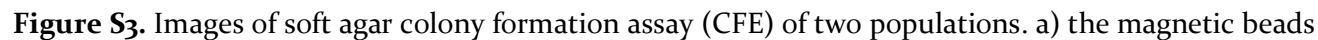
captured cells; b) the leftover populations. 2500 cells per well in 6-well plate were cultured in $0.35 \%$ soft agar for 2 weeks at $37{ }^{\circ} \mathrm{C}$ and $5 \% \mathrm{CO}_{2}$.
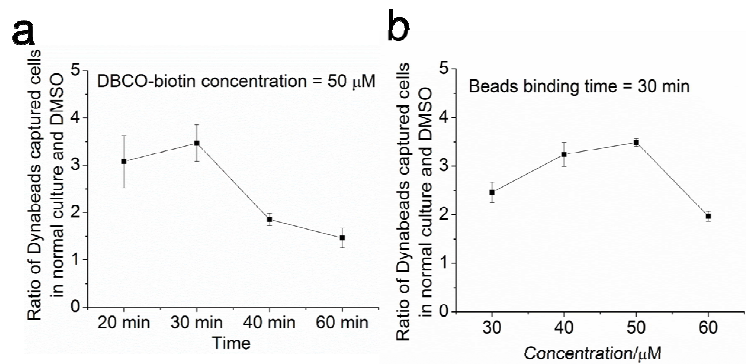

Figure S4. Ratio of the percentage of isolated cells from non-treated and 2\% DMSO treated HT-29 cells under different beads binding time (a) and concentration of DBCO-Biotin (b). 


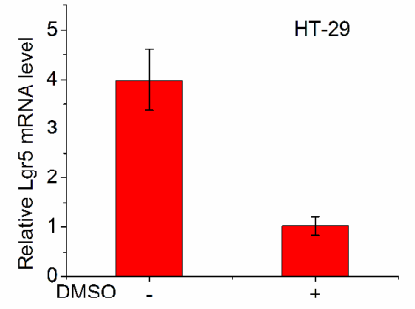

Figure S5. Relative Lgr5 mRNA levels of HT-29 cells without or with $2 \%$ DMSO. Cells are treated with Ac4ManNAz for 3 days. Gapdh was used as housekeeping gene. Error bars represent standard deviation from triplicate experiments. 